\title{
Phentolamine Mesylate as a Drug for Reversal of Local Anaesthesia - A Questionnaire Based Study
}

\author{
Chitharanjan Shetty ${ }^{1}$, Nikhita Devanathan ${ }^{2}$, Aditya Shetty ${ }^{3}$, Shishir Shetty ${ }^{4}$, Mithra N. Hegde ${ }^{5}$ \\ 1, 2, 3, 4, 5 Department of Conservative Dentistry and Endodontics, A.B. Shetty Memorial Institute of Dental \\ Sciences, Nitte Deemed to Be University, Mangalore, Karnataka, India.
}

\section{ABSTRACT}

\section{BACKGROUND}

Local anaesthesia being the most commonly administered drug in dentistry has its prolonged action for up to 3 - 5 hours due to the addition of vasoconstrictors. The extended periods of soft tissue anaesthesia due to the addition of these vasoconstrictors can cause other problems while speaking, drinking, and eating during which there is a higher risk of experiencing self-inflicted injuries to the tongue and lips. Phentolamine mesylate is a drug which helps in the reversal of action of local anaesthesia. Hence, the objective of this study was to provide a basic knowledge about this drug to the patient and assess the interest of the patient in taking the drug when given a choice.

\section{METHODS}

A questionnaire-based survey was conducted among 200 patients who required the administration of inferior alveolar nerve block for their dental treatment. This questionnaire contained basic information about Phentolamine Mesylate drug along with six questions where the patient was asked as to whether or not he / she was willing to take this drug for reversal of the effect of local anaesthesia and specific reason for its usage.

\section{RESULTS}

Among 200 patients, 122 patients wanted to reverse the effect of local anaesthesia by the use of the phentolamine mesylate drug. Amongst these patients, 93 wanted to take the drug orally initially and upon informing that the injection will be given in the previously anaesthetized area, 91 patients preferred to take an injection of the drug.

\section{CONCLUSIONS}

Majority of patients surveyed in this study wanted to reverse the effect of local anaesthesia by taking phentolamine mesylate drug. However, there is a need to increase the awareness of the type of drug used and the importance of administration of the same.

\section{KEY WORDS}

Phentolamine Mesylate, Local Anaesthesia, Questionnaire-Based Study, Soft Tissue Anaesthesia, Inferior Alveolar Nerve Block, Reversal of Anaesthesia
Corresponding Author: Dr. Nikhita Devanathan. Department of Conservative Dentistry and Endodontics, A. B. Shetty Memorial Institute of Dental Sciences, Nitte Deemed to Be University, Mangalore, Karnataka, India. E-mail: dnikhita27@gmail.com

DOI: $10.14260 /$ jemds/2020/708

How to Cite This Article:

Shetty $C$, Devanathan $N$, Shetty $A$, et al. Phentolamine mesylate as a drug for reversal of local anaesthesia - a questionnaire based study. J Evolution Med Dent Sci 2020;9(43):3227-3230, DOI: $10.14260 /$ jemds $/ 2020 / 708$

Submission 09-07-2020,

Peer Review 17-09-2020,

Acceptance 23-09-2020,

Published 26-10-2020.

Copyright (C) 2020 Chitharanjan Shetty et al. This is an open access article distributed under Creative Commons Attribution License [Attribution 4.0 International (CC BY 4.0)] 


\section{BACKGROUND}

Local anaesthetics are one of the most frequently administered drugs in dentistry. They have been shown to be extremely safe and effective for both the control and prevention of pain occurring during or following various invasive procedures. ${ }^{1}$ The time for which the local anaesthesia acts is one of the important criterions to be considered during the selection of local anaesthesia. It should have a rapid onset of action, with duration sufficient to complete the dental procedure comfortably. Based on their duration of action, these local anaesthetics can be broadly divided into shortacting, intermediate-acting and long-acting. The average duration of a dental treatment is about 47 minutes, thus an intermediately acting local anaesthetic can be used for these procedures. The duration of action of local anaesthetics also depends upon various other factors like the accuracy with which the drug has been administered, the status of the tissue at the site of injection and the technique used for administration.

A vasoconstrictor is often given in combination with these anaesthetics to compensate for its vasodilatory effects. They decrease the systemic plasma levels of these drugs and also achieve local ischemia, thus prolonging its effect. ${ }^{2}$ These vasoconstrictors also delay the absorption of local anaesthetic thus providing a degree of protection from its various systemic toxic effects. In spite of the above-mentioned function of these anaesthetics, patients often feel uncomfortable or disturbed due to other paucities caused during speaking, drinking, and eating during which there is a higher risk of experiencing selfinflicted injuries to the tongue and lips. The local anaesthetic agents, especially the drugs used for routine dental procedures which last for an hour or less and with minimal production of post-procedural pain have a few shortcomings, amongst which the most common being the residual soft tissue anaesthesia (numbness of the lip and tongue) lasting for a minimum of 3 to 5 hours. The anaesthesia to the tongue lasts longer than the lip anaesthesia in cases of mandibular block injections, increasing the incidence of self-inflicted injuries. ${ }^{3}$

Post treatment, in adult dental patients, the complaints concerning the residual anaesthesia can be in three aspects of their daily activities perceptual, sensory and functional which mainly include the problems experienced during eating and drinking, speaking and smiling, plus a fear of drooling in public. Whereas, in children, this prolonged soft tissue anaesthesia can lead to self-inflicting injuries to the lips, tongue, and cheeks. ${ }^{4,5}$

To overcome these effects of local anaesthesia, a nonselective alpha ( $\alpha_{1}$ and $\alpha_{2}$ ) adrenergic blocking agentPhentolamine Mesylate (PM) can be used. The primary development of this drug was for the treatment of hypertension due to its pronounced effect of vasodilation. It was also used for the treatment of patients with pheochromocytoma and dermal necrosis. The mechanism by which PM reverses the effect of local anaesthesia is by antagonizing the vasoconstricting property of epinephrine, resulting in systemic absorption of the local anaesthetic.6,7 It is known to have a structural similarity with its agonist epinephrine, which enables it to bind to the receptor site but does not cause the receptor activation. ${ }^{8}$ The phentolamine mesylate is an antagonist for the epinephrine added to the local anaesthetic but not to the local anaesthetic itself. Thus, its effect cannot be evaluated in anaesthesia administered without vasoconstrictors.

Based on this property of vasodilatory effect, a dental local anaesthesia reversal agent was approved by the US Food and Drug Administration in the year 2008 and was marketed in the name of OraVerse (Septodont). This product was first used in Germany in the year 2011 for reversal of soft tissue anaesthesia of lip and tongue. For the reversal of anaesthesia administered for dental procedures, only $0.4 \mathrm{mg}$ of phentolamine mesylate drug in $1.7 \mathrm{ml}$ of packaged solution is packed into the dental cartridge. This is then injected into the same location with the same volume of drug and injection technique which was used for the previous injection of local anaesthesia after the completion of the desired dental procedure. ${ }^{9}$ The average reduction in time for the recovery of the lip and tongue numbness is normally an hour for phentolamine mesylate drug. This drug is not advised to be used in children of age 6 years and less or children weighing less than $15 \mathrm{kgs}$ due to the lack of appropriate clinical trials.

The present study aims at providing a basic knowledge about this drug to the patient and assessing the interest of the patient in taking the drug for reversal of extended soft tissue anaesthesia when given a choice.

\section{METHODS}

This cross sectional, self-administered and structured closed ended questionnaire-based survey was conducted among 200 patients who reported to the Department of Conservative Dentistry and Endodontics, who required the administration of inferior alveolar nerve block for their dental treatment. A questionnaire containing basic information of the drug including its indications, contraindications and adverse effects was given to the patient. This questionnaire consisted of six questions where the patient was asked whether or not, he / she was willing to take the drug for the reversal of the effect of local anaesthesia and specific reason for the usage of this drug.

\section{RESULTS}

Out of the 200 patients who were administered the local anaesthetic drug in their previous appointments, 21 patients reported that the effect of the local anaesthetic lasted for more than 3 hours. The effect lasted for 2 - 3 hours in 98 patients. And for the rest of the patients the effect lasted for 2 hours or less. [Table 1].

\begin{tabular}{|ccc|}
\hline Duration & Frequency & Percentage (\%) \\
$0-1 \mathrm{hr}$. & 17 & 8.5 \\
$1-2 \mathrm{hr}$. & 64 & 32.0 \\
$2-3 \mathrm{hr}$ & 98 & 49.0 \\
More than $3 \mathrm{hr}$. & 21 & 10.5 \\
Total & 200 & 100.0 \\
\hline Table 1. Duration of Action of Local & Anaesthesia \\
\hline
\end{tabular}

Out of the 200 patients, 169 patients who were administered the local anaesthesia described that their daily activities were altered by its effect [Table 2] and the most commonly affected function was talking (115) followed by smiling / facial expression (22), chewing (17) and others (15) [Table 3]. 


\begin{tabular}{|ccc|}
\hline & Frequency & Percentage (\%) \\
Yes & 169 & 84.5 \\
No & 31 & 15.5 \\
Total & $\mathbf{2 0 0}$ & $\mathbf{1 0 0 . 0}$ \\
\hline Table 2. Did the Effect of Local Anaesthesia Alter the Daily Activities \\
\hline
\end{tabular}

\begin{tabular}{|ccc|}
\hline & Frequency & Percentage (\%) \\
\hline Smiling / Facial Expressions & 22 & 13.0 \\
Chewing & 17 & 10.1 \\
Talking & 115 & 68.0 \\
Others & 15 & 8.9 \\
Total & 169 & 100.0 \\
\hline Table 3. Most Commonly Affected Function \\
\hline
\end{tabular}

Out of 169 patients, 122 (72\%) wanted to reverse the effect of local anaesthesia soon after the dental procedure.

Amongst 122 patients, 93 preferred oral route of drug administration rather than an injection to reverse the effect of local anaesthesia [Table 4] and when asked whether they would take another injection at the previously anaesthetized site, 91 patients were willing to take another injection [Table 5].

\begin{tabular}{|ccc|}
\hline & Frequency & Percent \\
\hline Injection & 29 & 23.8 \\
Oral Medication & 93 & 76.2 \\
Total & $\mathbf{1 2 2}$ & $\mathbf{1 0 0 . 0}$ \\
\hline Table 4. Preferred Mode for Reversal of Effect of Local Anaesthesia \\
\hline
\end{tabular}

\begin{tabular}{|ccc|}
\hline & Frequency & Percent \\
Yes & 91 & 74.6 \\
No & 31 & 25.4 \\
Total & 122 & 100.0 \\
\hline Table 5. When the Area is already Anaesthetized, Would You \\
Like to Take another Injection of Phentolamine Mesylate \\
Drug to Reverse the Action of Local Anaesthesia
\end{tabular}

\section{DISCUSSION}

The present study is a cross sectional, self-administered, close ended questionnaire-based survey which was conducted to assess the interest of patients taking phentolamine mesylate drug for the reversal of the effect of local anaesthesia. A close ended questionnaire was used in this study as it requires less time to answer; the communication skills of the respondent is less critical and the respondent finds it easier to answer.

Typically, teeth recover more quickly than surrounding bone and soft tissues such as lips and tongue. This soft tissue anaesthesia (STA) lasts approximately 3 to 5 hours and is frequently substantially longer than the time necessary for completion of many routine dental procedures. ${ }^{10}$ In this study, amongst the 200 patients surveyed, 98 patients said that the effect of local anaesthesia lasted for up to 2 to 3 hours after the completion of the procedure, and for 21 patients it lasted for more than 3 hours. The duration of action of local anaesthesia depends upon the type of anaesthetic drug used and also on the method of administration of the drug. Lidocaine hydrochloride with epinephrine (1:100000) is the most commonly used local anaesthetic in the dental practice with a duration of action of 180 - 300 minutes for STA when administered as a nerve block. ${ }^{11}$ Whereas, the median time of recovery for normal lip function after administration of phentolamine mesylate is around one hour. Rafique et al. conducted a study in which they found that $86 \%$ of patients undergoing dental treatment which includes administration of local anaesthesia reported moderate dislike of postoperative numbness and $14 \%$ of patients showed high dislike. A few of these patients apart from having discomfort physically, also refrained from many social activities due to the impairment of sensory function. ${ }^{12}$

According to this study, $68 \%$ of the patients complained of difficulty while talking, which is the most common daily activity of an individual. The lingual nerve which supplies the anterior two thirds of the tongue is also anaesthetized to carry out dental treatment. This is one of the main reasons for the patients to experience impaired speech. The second most common daily activity affected by the administration of local anaesthesia is smiling and alteration in facial expressions (13 $\%$ ). These are considered to be the most common unwanted side effects of soft tissue anaesthesia in adult patients and lip biting to be the most common in paediatric patients. In order to reverse these effects, Laviola, et al. in their study administered PM immediately after completion of dental treatment where local anaesthesia was administered prior to the treatment. The results of their study showed one-hour reduction in reversal time of normal lip sensation. They also found that this reduction had no significant relationship with the age or sex of the patient, and also the type of treatment. 5

In another study by Tavares et al., there was a $55.6 \%$ reduction in the reversal time of normal lip sensation and 60 $\%$ reduction in the reversal time of normal tongue sensation in paediatric patients. ${ }^{13}$

Due to the presence of these alterations in the daily activities, 122 out of 169 (72\%) patients in this study wanted to reverse the effect of soft tissue anaesthesia. In a survey conducted by College et al. on paediatric patients, $13 \%$ of inferior alveolar nerve blocks were associated with patients inadvertently biting their lips. When viewed according to the age group, the trauma seen was as $18 \%$ (< 4 years), $16 \%$ (4 7 years), $13 \%$ ( $8-11$ years) and $7 \%$ (> 12 years). ${ }^{14}$

From a patient's perspective, using an injection is one of the painful methods of drug administration as it includes the piercing of the soft tissue. Oral route of drug administration is preferred over using injection by most of the patients. Due to the same reasons, 93 out of 122 (76.2\%) patients preferred oral medication over injection as the mode of administration of phentolamine mesylate.

The PM injection is usually administered with the same dosage as the local anaesthesia used prior to the commencement of the treatment and at the same site of the previous injection. As this area will already be anaesthetized, the patient will not be experiencing any pain during the administration of the second injection containing PM. After explaining the above-mentioned procedure and assuring the painless administration of the drug, 91 out of 122 patients agreed to take another injection to reverse the effect of local anaesthesia. Patient's psychological state regarding the perception of pain during the dental treatment plays a major role in deciding the suitable treatment plan.

\section{CONCLUSIONS}

A majority of dental treatments is not traumatic and does not require the effect of local anaesthesia to last for a longer period of time. Faster recovery of normal sensation of lip and tongue, as well as a faster return of the normal ability to smile, speak, drink, and to refrain from drooling in public and inflicting selfinjury are highly desirable. In this study, majority of the 
patients surveyed desired to reverse the effect of local anaesthesia on the soft tissues using the drug orally. Awareness about drugs used for the reversal of local anaesthesia should be created to prevent the adverse effects of prolonged residual soft tissue anaesthesia.

Data sharing statement provided by the authors is available with the full text of this article at jemds.com.

Financial or other competing interests: None.

Disclosure forms provided by the authors are available with the full text of this article at jemds.com.

\section{REFERENCES}

[1] Malamed SF. Local anaesthesia. J Calif Dent Assoc 1998;26(9):657-60.

[2] Daubländer M, Liebaug F, Niedeggen G, et al. Effectiveness and safety of phentolamine mesylate in routine dental care. J Am Dent Assoc 2017;148(3):149-56.

[3] Malamed SF. Phentolamine mesylate for the reversal of residual soft-tissue anaesthesia. Acad Dent Ther Stomatol 2008.

[4] Hersh EV, Moore PA, Papas AS, et al. Reversal of softtissue local anaesthesia with phentolamine mesylate in adolescents and adults. J Am Dent Assoc 2008;139(8):1080-93.

[5] Laviola M, McGavin SK, Freer GA, et al. Randomized study of phentolamine mesylate for reversal of local anaesthesia. J Dent Res 2008;87(7):635-9.
[6] Rhoney D, Peacock WF. Intravenous therapy for hypertensive emergencies, part 1. Am J Health Syst Pharm 2009;66(15):1343-52.

[7] Phentolamine mesylate for injection package insert http://www.bedfordlabs.com/BedfordLabsWeb/produc ts/inserts/PHT-P01.pdf.

[8] Malamed S. What's new in local anaesthesia. SAAD Dig 2009;25:4-14.

[9] Ora verse package insert. http://www.novalar.com/assets/pdf/package_inset_jan 09.pdf

[10] Hersh EV, Hermann DG, Lamp CJ, et al. Assessing the duration of mandibular soft tissue anaesthesia. J Am Dent Assoc 1995;126(11):1531-6.

[11] Malamed SF. Handbook of local anaesthesia. $5^{\text {th }}$ edn. St. Louis, Mo: Mosby 2004:293-294.

[12] Rafique S, Fiske J, Banerjee A. Clinical trial of an airabrasion/ chemomechanical operative procedure for the restorative treatment of dental patients. Caries Res 2003;37(5):360-4.

[13] Tavares M, Goodson JM, Studen-Pavlovich D, et al. Reversal of soft-tissue local anaesthesia with phentolamine mesylate in pediatric patients. J Am Dent Assoc 2008;139(8):1095-104.

[14] College C, Feigal R, Wandera A, et al. Bilateral versus unilateral mandibular block anaesthesia in a pediatric population. Pediatr Dent 2000;22(6):453-7. 